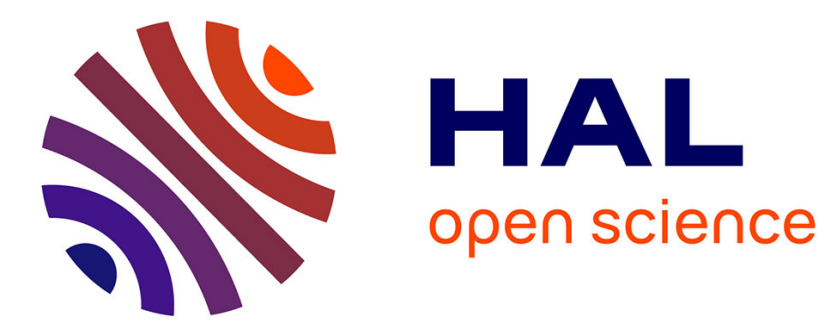

\title{
Observer design for a ternary distillation column with side stream
}

Chouaib Afri, Madiha Nadri, Pascal Dufour

\section{To cite this version:}

Chouaib Afri, Madiha Nadri, Pascal Dufour. Observer design for a ternary distillation column with side stream. 53rd IEEE Conference on Decision and Control (CDC), Dec 2014, Los Angeles, CA, United States. pp.6383-6388, 10.1109/CDC.2014.7040390 . hal-01264107

\section{HAL Id: hal-01264107 \\ https://hal.science/hal-01264107}

Submitted on 1 Feb 2016

HAL is a multi-disciplinary open access archive for the deposit and dissemination of scientific research documents, whether they are published or not. The documents may come from teaching and research institutions in France or abroad, or from public or private research centers.
L'archive ouverte pluridisciplinaire HAL, est destinée au dépôt et à la diffusion de documents scientifiques de niveau recherche, publiés ou non, émanant des établissements d'enseignement et de recherche français ou étrangers, des laboratoires publics ou privés. 
This document must be cited according to its final version which is published in a journal as:

C. Afri, M. Nadri, P. Dufour, "Observer design for a ternary distillation column with side stream", 53rd IEEE Conference on Decision and Control (CDC), Los Angeles, CA, USA, pp. 6383-6388, december 15-17, 2014.

DOI : 10.1109/CDC.2014.7040390

You downloaded this document from the CNRS open archives server, on the webpages of Pascal Dufour: http://hal.archives-ouvertes.fr/DUFOUR-PASCAL-C-3926-2008 


\title{
Observer Design for a Ternary Distillation Column with Side Stream
}

\author{
Chouaib Afri, Madiha Nadri Wolf and Pascal Dufour
}

\begin{abstract}
This paper investigates the problem of observer design to provide temperature and molar fraction spatial profiles in a ternary distillation column with side stream. Indeed, both temperature and molar fraction spatial profiles are required to be used in advanced controller design. Such an estimation problem is usually solved using an implicit observer which requires an optimization algorithm when a differential algebraic system is used. In this paper, we consider a realistic nonlinear differential algebraic equation based model validated experimentally. We propose an approach to design an explicit observer that reconstructs all the state variables with an asymptotic convergence. The observer is build as a coupling of two nonlinear observers in full interconnection. Finally, the performance of the proposed observer is illustrated using a model of a ternary distillation column with side stream.
\end{abstract}

\section{INTRODUCTION}

The industry is still looking for better strategies for improving purity control in distillation columns, while optimizing installation costs and energy. The constraints imposed on such system are: the high cost of the measures and the nonlinear coupling between physical quantities. The most common controllers in the industrial world are PID, which tuning may be based on a simple model to describe the process. Their decentralized control loop feature results in high energy consumption and poor control performances. Hence, advanced controllers are needed, using a model based state estimation. The model used here is based on the total and partial material balances and the vapor liquid equilibrium (VLE) [1]. In this work, we do not consider the classical assumption of ideal mixture: the components to be separated can have a completely different chemical structures (such as oil and water) resulting in forces of attraction or repulsion between adjacent molecules of different types. This phenomenon is reflected by the activity coefficients which are functions of the component concentrations of the liquid mixture. A few works have considered the nonideal case [2]. Unlike to the classical models based on LEWIS assumptions [3], which can be represented as an ordinary differential equations (ODE) system, the model considered here is closer to the real behaviour and is a set of nonlinear differential algebraic equations (DAE). Using algebraic methods, observer design for linear DAE systems has widely been investigated in the literature (see for instance [4], [5], [6], [7] and the references herein). [8] extends the Kalman filter for discrete time systems (the proposed Kalman filter is also an implicit system). The observer synthesis for nonlinear DAE systems is still an open problem. The most

C. Afri, M. Nadri Wolf and P. Dufour are with the Université de Lyon, F-69622, Lyon, France - Université Lyon 1, Villeurbanne, France - CNRS, UMR 5007, LAGEP, France. $\{$ nadri\}@lagep.univ-lyon 1 .fr common approaches consist in characterizing this DAE class which can be transformed by a diffeomorphism to an ODE system and for which an observer can be designed [9], [10], [11]. The observer for the original system is an implicit system obtained by using the pullback map.

Beside these geometric approaches, other approaches gave implicit observers whose practical implementation usually requires the use of optimization techniques to solve at each time $t$ the algebraic constraints associated to the state manifold together with an ODE numerical method (see for instance [12], [13], [14] and [15]). Such methods have been shown to lack robustness when solving large scale complex systems or systems with fast dynamics. Another interesting approach is presented in [16]. In their work, the authors used the algebraic constraints as additional output and gave an explicit observer of higher dimension.

This work aims to design an explicit state observer based on a realistic experimentally validated nonlinear DAE model. The outline of the paper is as follows. In section 2, we summarize the model development for a ternary distillation column with side stream. In section 3, we synthesize an explicit observer for a class of nonlinear DAE systems which contains most models of distillation columns. Finally, the usefulness of this approach is illustrated using only four molar fraction measurements to estimate both temperature and molar fractions spatial profiles.

\section{STATE MODEL OF A TERNARY DISTILLATION COLUMN WITH SIDE STREAM}

The considered process is a ternary distillation column with side stream (figure 1) separating a liquid ternary mixture of BTX (Benzene, Toluene and o-Xylene, considered as the component 1, 2 and 3 respectively) [17]. The column consists of (n-2) theoretical trays, a boiler (tray $n$ )and a condenser (tray 1). Using the material balance, we get the DAE (1), where, $j=1,2,3$ is the component index, $i=1, \ldots, n$ is the tray index, $N_{i}$ is the molar retention in the tray $i, x_{j, i}$ the liquid phase molar fraction of component $j$ in the tray $i, y_{j, i}$ is the vapour phase molar fraction of component $j$ in the tray $i, T_{i}$ is the stage temperature in the tray $i, Z_{j, l}$ is the feed flow molar fraction of component $j$ in the tray $l, F$ is the molar feed rate in the tray $l, S$ is the molar flow rate extracted from tray $s, L$ is the molar flow rate of reflux and $V_{f}$ is the molar vapour flow rate. Fractions of the third component is calculated from the equation of matter conservation (2). 


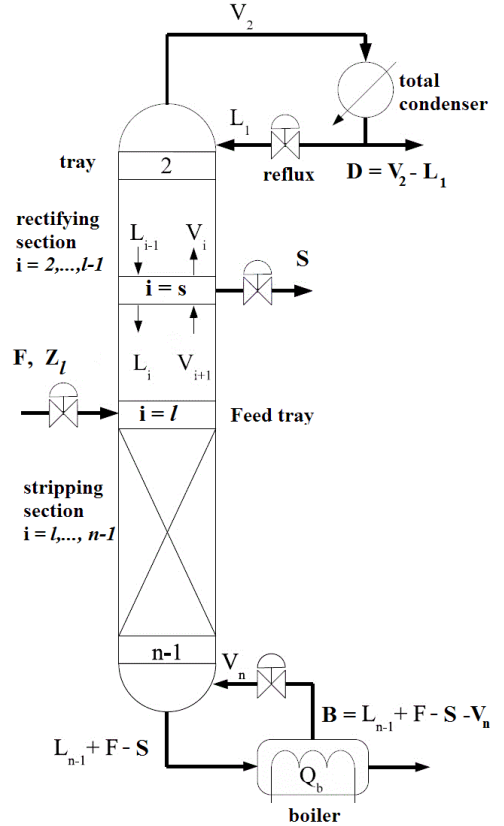

Fig. 1. Scheme of a distillation column with side stream.

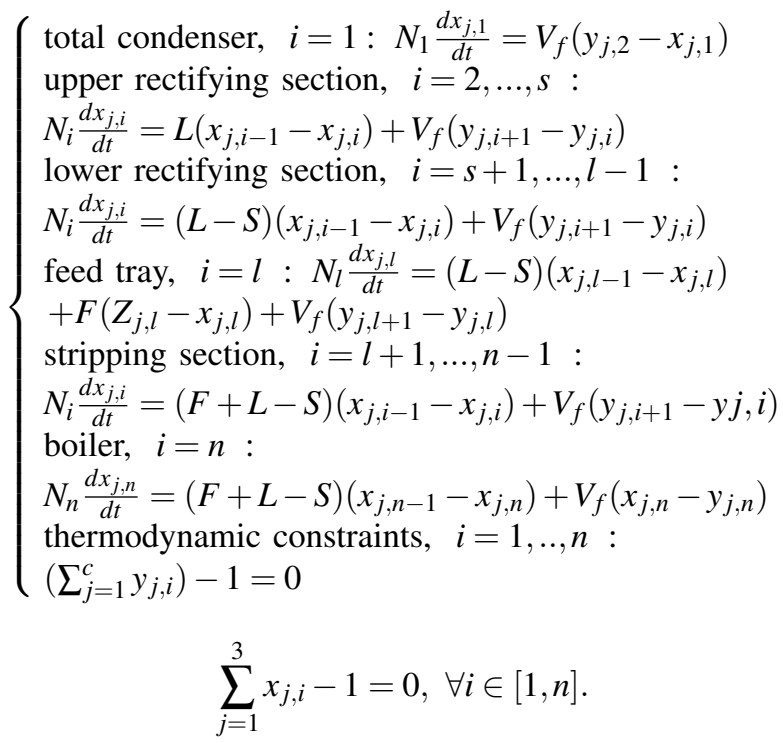

From now, based on (2), we set that $x_{3, i}$ is a function of $x_{1, i}$ and $x_{2, i}$. From the VLE and the Antoine equation [17], [1] we get:

$$
\left\{\begin{array}{l}
\text { rectifying section: } \quad 1 \leq i<l \\
y_{j, i}=\frac{\gamma_{j, i}\left(x_{1, i}, x_{2, i}, T_{i}\right)}{P_{T} e_{i}^{l}}\left(\left(x_{j, i}-\left(1-e_{i}^{l}\right)\left(x_{j, i-1}\right)\right) 10^{a_{j}-\frac{b_{j}}{T_{i}+c_{j}}}\right) \\
\text { stripping section: } \quad l \leq i \leq n-1 \\
y_{j, i}=\left(1-e_{i}^{v}\right) y_{j, i+1}+\frac{e_{i}^{v} \gamma_{j, i}\left(x_{1, i}, x_{2, i}, T_{i}\right)}{P_{b_{j}}}\left(x_{j, i} 10^{a_{j}-\frac{b_{j}}{T_{i}+c_{j}}}\right) \\
\text { boiler: } \quad y_{j, n}=\frac{1}{P_{T}}\left(x_{j, n} 10^{a_{j}-\frac{b_{j}}{T_{n}+c_{j}}} \gamma_{j, n}\left(x_{1, i}, x_{2, i}, T_{n}\right)\right)
\end{array}\right.
$$

where, $\gamma_{j, i}$ is the activity coefficient that represents the non ideality of mixture, $e_{i}^{l}, e_{i}^{v}$ are respectively the rectifying and stripping efficiencies [1], and $P_{T}$ the internal pressure. The $\gamma_{j, i}$ 's and Antoine parameters $\left(a_{j}, b_{j}\right.$ and $\left.c_{j}\right)$ are obtained from binary mixtures experimental data of the three components BTX [17]. This choice is the best in terms of accuracy compared to the ternary experimental data [2]. The difference between the classical models [3] and this one is that the mixture here is not ideal. Consequently, $\gamma_{j, i}$ is a function of liquid molar fractions $x_{j, i}$ and temperature $T_{i}$. We divide the process to a rectifying section and a stripping section, with exponents ${ }^{1}$ and ${ }^{2}$ respectively, and introduce the following notations :

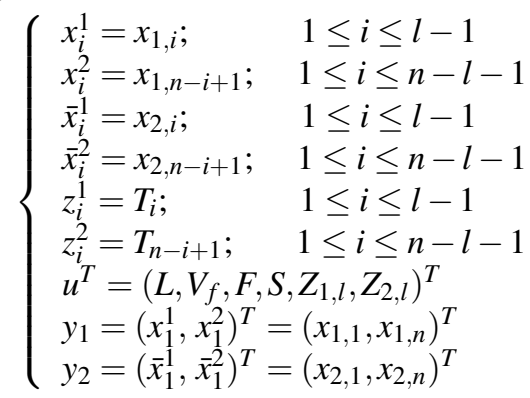

where $y_{1}$ contains the top and bottom measures of light component (Benzene), $y_{2}$ contains the top and bottom measures of intermediate component (Toluene). $F, S, Z_{1, l}$ and $Z_{2, l}$ are the unknown input disturbances, $V_{f}, L$ are manipulated inputs of the input vector $u . x$ and $\bar{x}$ are the state variables. Using (4), the system (1)-(3) belongs to the following fully interconnected systems [20]:

$$
\begin{aligned}
& (\Sigma)\left\{\begin{array}{l}
\dot{x}(t)=f(x(t), \bar{x}(t), z(t), u(t)) \\
\varphi(x(t), \bar{x}(t), z(t))=0 \\
y_{1}(t)=h(x)=\left[x_{1}^{1}(t), x_{1}^{2}(t)\right]^{T}
\end{array}\right. \\
& (\bar{\Sigma})\left\{\begin{array}{l}
\dot{\bar{x}}(t)=\bar{f}(x(t), \bar{x}(t), z(t), u(t)) \\
\varphi(x(t), \bar{x}(t), z(t))=0 \\
y_{2}(t)=\bar{h}(\bar{x})=\left[\bar{x}_{1}^{1}(t), \bar{x}_{1}^{2}(t)\right]^{T}
\end{array}\right.
\end{aligned}
$$

where,

$$
\begin{aligned}
& x=\left(x_{1}^{1}, . ., x_{l-1}^{1}, x_{1}^{2}, . ., x_{n-l+1}^{2}\right)^{T} ; \bar{x}=\left(\bar{x}_{1}^{1}, . ., \bar{x}_{l-1}^{1}, \bar{x}_{1}^{2}, . ., \bar{x}_{n-l+1}^{2}\right)^{T} \\
& f=\left[\begin{array}{l}
f^{1}(\bar{x}, x, z, u) \\
f^{2}(x, \bar{x}, z, u)
\end{array}\right] ; \bar{f}=\left[\begin{array}{l}
\bar{f}^{1}(\bar{x}, x, z, u) \\
\bar{f}^{2}(x, \bar{x}, z, u)
\end{array}\right] ; \varphi=\left[\begin{array}{l}
\varphi^{1}\left(x^{1}, \bar{x}^{1}, z^{1}\right) \\
\varphi^{2}\left(x^{2}, \bar{x}^{2}, z^{2}\right)
\end{array}\right]
\end{aligned}
$$

$f, \bar{f}$ and $\varphi$ are $\mathscr{C}^{1}$ functions with respect to their arguments and satisfy the following triangular structure:

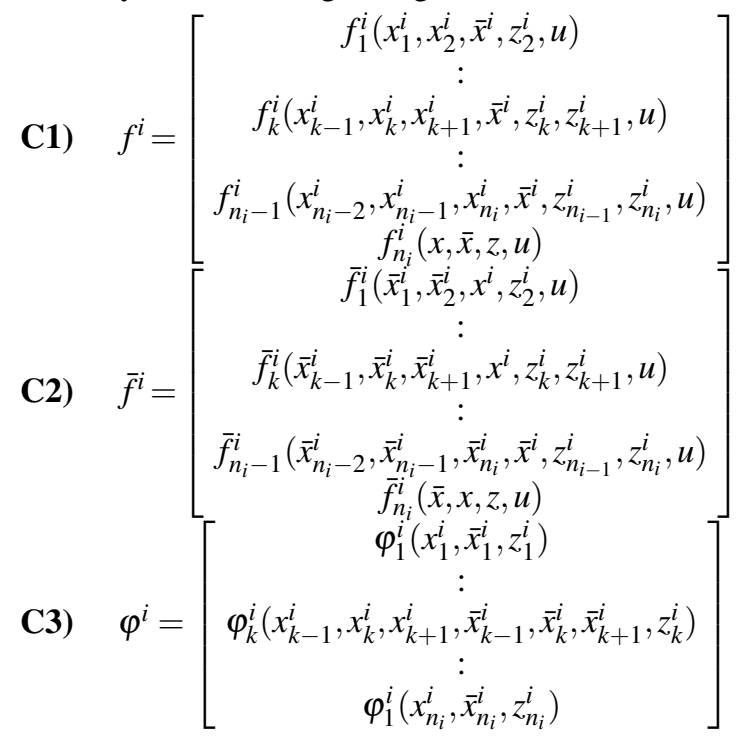


where $n_{1}=l-1$, and $n_{2}=n-l+1$.

\section{EXPLICIT OBSERVER DESIGN FOR THE FULLY INTERCONNECTED IMPLICIT SYSTEMS}

The system under consideration $(\Sigma, \bar{\Sigma})$ belongs to the class of implicit systems written in the following compact format:

$$
\begin{cases}\dot{x}(t)=f(x(t), \bar{x}(t), z(t), u(t)) & x \in \mathbb{R}^{n}, \quad u \in U \\ \dot{\bar{x}}(t)=\bar{f}(x(t), \bar{x}(t), z(t), u(t)) & \bar{x} \in \mathbb{R}^{n} \\ \varphi(x(t), \bar{x}(t), z(t))=0 & z \in \mathbb{R}^{d} \\ y(t)=[h(x(t)) \bar{h}(\bar{x}(t))]^{T} & y \in \mathbb{R}^{p},\end{cases}
$$

where $U$ is a bounded open subset of $\mathbb{R}^{m},(x(t), \bar{x}(t), z(t)) \in$ $\mathbb{R}^{n} \times \mathbb{R}^{n} \times \mathbb{R}^{d}$ is the state vecteur, $f, \bar{f}, h$ and $\bar{h}$ are assumed to be sufficiently smooth with respect to their arguments. Let $\mathscr{M}$ denotes the constraints set,

$$
\mathscr{M}=\left\{(x, \bar{x}, z) \in \mathbb{R}^{n} \times \mathbb{R}^{n} \times \mathbb{R}^{d} \text {, s.t. } \varphi(x, \bar{x}, z)=0\right\}
$$

In [18] the authors consider a class of implicit systems of the form (5) and gave an high gain explicit observer. Based on this result, we will show that the following system (9) is an asymptotic observer for system (7).

$$
\left\{\begin{aligned}
\dot{\hat{x}}(t) & =\hat{f}(\hat{x}(t), \hat{\bar{x}}(t), \hat{z}(t), g(t), h(\hat{x}(t)), u(t)) \\
\dot{\hat{x}}(t) & =\hat{\hat{f}}(\hat{x}(t), \hat{\bar{x}}(t), \hat{z}(t), \bar{g}(t), \bar{h}(\hat{\bar{x}}(t)), u(t)) \\
\dot{\hat{z}}(t) & =-\left(\left.\frac{\partial \varphi}{\partial z}\right|_{(\hat{x}, \hat{z}, \hat{x})}\right) \\
& \times\left(\left[\left.\frac{\partial \varphi}{\partial x}\right|_{(\hat{x}, \hat{z})},\left.\frac{\partial \varphi}{\partial \bar{x}}\right|_{(\hat{x}, \hat{z})}\right][\dot{\hat{x}}, \dot{\hat{x}}]^{T}+\Lambda \varphi(\hat{x}, \hat{\bar{x}}, \hat{z})\right) \\
\dot{g}(t) & =G\left(g(t), y_{1}(t), u(t)\right) \\
\dot{\bar{g}}(t) & =\bar{G}\left(\bar{g}(t), y_{2}(t), u(t)\right),
\end{aligned}\right.
$$

where, $\Lambda$ is a $d \times d$ symmetric positive definite matrix (SPD), $g$ and $\bar{g}$ in $\mathbb{R}^{n \times p}$ are the observer gain functions defined in the next sections. The following assumptions are considered.

Assumption $\mathscr{A}$ 1): There exists $\varepsilon_{0}>0$ such that $\frac{\partial \varphi}{\partial z}$ has a full rank on the tubular neighborhood $\Omega_{0}=\{(x, \bar{x}, z) \in$ $\left.\mathbb{R}^{n+n+d} ;\|\varphi(x, \bar{x}, z)\|<\varepsilon_{0}\right\}$, and there exists a function $\sigma$ of class $\mathscr{K}_{\infty}$ such that:

i) The following map:

$$
\begin{array}{cccc}
\Phi: \quad \Omega_{0} & \longrightarrow & \mathbb{R}^{n+n} \times J \\
& (x, \bar{x}, z) & \longrightarrow & (x, \bar{x}, \varphi(x, \bar{x}, z))
\end{array}
$$

is a diffeomorphism, where $J$ is an open subset of $\mathbb{R}^{d}$.

ii) $\forall(x, \bar{x}, z) \in \Omega_{0},\|\varphi(x, \bar{x}, z)\| \geq \sigma\left(\left\|z-z^{*}\right\|\right)$

$z^{*}$, is the solution of $\varphi(x, \bar{x}, z)=0$.

The next hypothesis guaranties the existence of an implicit observer:

\section{Assumption $\mathscr{A}$ 2):}

1) There exists $\mathscr{U} \subset L^{\infty}\left(\mathbb{R}^{+}, U\right)$, such that for every $u \in$ $\mathscr{U}$; for every $(g(0)),(\bar{g}(0)) \in O$, the trajectory $g($.$) and$ $\bar{g}($.$) are bounded.$

2) There exist positive definite functions $V(t, e), \bar{V}(t, \bar{e})$, $W(e)$ and $\bar{W}(\bar{e})$ defined on $\mathbb{R}^{+} \times \mathbb{R}^{n}$ such that for every $u \in \mathscr{U}$, we have:

i) $\forall(x, \bar{x}, z) \in \mathscr{M} ; \forall(\hat{x}, \hat{\bar{x}}, \hat{z}) \in \mathscr{M} ; \forall t \geq 0$; we have

$$
\begin{aligned}
\frac{\partial V}{\partial t}(t, e)+ & \frac{\partial V}{\partial e}(t, e)\left(\hat{f}\left(e+x, \hat{\bar{x}}, z^{*}, g, h(\hat{x}), u\right)\right. \\
& -f(x, \hat{\bar{x}}, z, u)) \leq-W(e) \\
\frac{\partial \bar{V}}{\partial t}(t, \bar{e})+ & \frac{\partial \bar{V}}{\partial \bar{e}}(t, \bar{e})\left(\hat{f}\left(\hat{x}, \bar{e}+\bar{x}, z^{*}, \bar{g}, \bar{h}(\hat{\bar{x}}), u\right)\right. \\
& -f(\hat{x}, \bar{x}, z, u)) \leq-\bar{W}(\bar{e})
\end{aligned}
$$

where $e=\widehat{x}-x$ and $\bar{e}=\widehat{\bar{x}}-\bar{x}$.

ii) There exist constants $a \geq 0, \alpha>0$ and $\bar{\alpha}>0$; such that $\forall e, \bar{e} ; \forall t \geq 0$

$$
\left\|\frac{\partial V}{\partial e}(t, e)\right\|+\left\|\frac{\partial \bar{V}}{\partial \bar{e}}(t, \bar{e})\right\| \leq \alpha W(e)+\bar{\alpha} \bar{W}(\bar{e})
$$

iii) $\exists \alpha_{1}>0, \alpha_{2}>0 ; \forall(x, \bar{x}) \in \mathbb{R}^{n} \times \mathbb{R}^{n} ; \forall(e, \bar{e}) \in$ $\mathbb{R}^{n} \times \mathbb{R}^{n} ; \forall t \geq 0$

$$
\begin{array}{r}
\left\|\frac{\partial V}{\partial e}(f(x, \bar{x}+\bar{e}, z, u)-f(x, \bar{x}, z, u))\right\| \\
\leq \alpha_{1} \sqrt{W(e)} \sqrt{\bar{W}(\bar{e})}, \\
\left\|\frac{\partial \bar{V}}{\partial \bar{e}}(\bar{f}(x+e, \bar{x}, z, u)-\bar{f}(x, \bar{x}, z, u))\right\| \\
\leq \alpha_{2} \sqrt{W(e)} \sqrt{\overline{\bar{W}(\bar{e})}},
\end{array}
$$$$
0<\alpha_{1}+\alpha_{2} \leq 2
$$

Finally, we consider the Lipschitz condition:

Assumption $\mathscr{A}$ 3): There exists a class $\mathscr{K}_{\infty}$ functions $\lambda_{1}($. and $\lambda_{2}($.$) such that, \forall\left(\widehat{x}, \widehat{\bar{x}}, \widehat{z}, z^{*}(\widehat{x}, \widehat{\bar{x}})\right) \in \Omega_{0} ; \forall g$, we have: $\left\|\hat{f}(\widehat{x}, \widehat{\bar{x}}, \widehat{z}, g, h(\hat{x}), u)-\hat{f}\left(\widehat{x}, \widehat{\bar{x}}, z^{*}, g, h(\hat{x}), u\right)\right\| \leq \lambda_{1}\left(\left\|\widehat{z}-z^{*}\right\|\right)$ $\left\|\hat{f}(\widehat{x}, \hat{\bar{x}}, \widehat{z}, \bar{g}, \bar{h}(\hat{\bar{x}}), u)-\hat{\bar{f}}\left(\widehat{x}, \widehat{\bar{x}}, z^{*}, \bar{g}, \bar{h}(\hat{\bar{x}}), u\right)\right\| \leq \lambda_{2}\left(\left\|\widehat{z}-z^{*}\right\|\right)$

Now consider $\Lambda$ defined as above, and let $\varepsilon_{\Lambda}>0$ be such that $\Omega_{\Lambda}=\left\{(x, \bar{x}, z) \in \mathbb{R}^{n} \times \mathbb{R}^{n} \times \mathbb{R}^{d} ; \varphi(x, \bar{x}, z)^{T} \Lambda \varphi(x, \bar{x}, z)<\right.$ $\left.\varepsilon_{\Lambda}\right\} \subset \Omega_{0}$. Noticing that $\Omega_{\Lambda}=\Omega_{0}$ if $\Lambda=I$ and $\varepsilon_{\Lambda}=\varepsilon_{0}$.

Using these notations, we can state our main result as follow.

Theorem 1: Under assumptions $\mathscr{A}$ 1), $\mathscr{A}$ 2) and $\mathscr{A}$ 3) , system (9) forms an asymptotic explicit observer for system (7).

Proof: $\quad \operatorname{Let}(x(t), \bar{x}(t), z(t))$ and $(\hat{x}(t), \hat{\bar{x}}(t), \hat{z}(t))$ be the trajectories of systems (7) and (9) respectively. From (9) we can get:

$$
\frac{d\|\varphi(\hat{x}(t), \hat{\bar{x}}(t), \hat{z}(t))\|^{2}}{d t}=-2(\varphi(\hat{x}, \hat{\bar{x}}, \hat{z}))^{T} \Lambda(\varphi(\hat{x}, \hat{\bar{x}}, \hat{z}))
$$

From assumption $\mathscr{A} \mathbf{1}), \Phi$ is a diffeomorphism from $\Omega_{0}$ into $\mathbb{R}^{n+n} \times J$, so there exists a unique solution $z^{*}(t)$, such that $\varphi\left(\hat{x}(t), \hat{\bar{x}}(t), z^{*}(t)\right)=0$. Let $e(t)=\hat{x}(t)-x(t), \bar{e}(t)=\hat{\bar{x}}(t)-$ $\bar{x}(t)$ be the estimation errors of the observer. So from (7) and (9) we can get:

$$
\begin{aligned}
\dot{e}(t)+ & \dot{\bar{e}}(t)=\hat{f}\left(\hat{x}, \hat{\bar{x}}, z^{*}, g, h(\hat{x}), u\right)-f(x, \hat{\bar{x}}, z, u) \\
& +\hat{\bar{f}}\left(\hat{\bar{x}}, \hat{\bar{x}}, z^{*}, \bar{g}, h(\hat{\bar{x}}), u\right)-\bar{f}(\hat{x}, \bar{x}, z, u) \\
& +f(x, \hat{\bar{x}}, z, u)-f(x, \bar{x}, z, u) \\
& +\bar{f}(\hat{x}, \bar{x}, z, u)-\bar{f}(x, \bar{x}, z, u) \\
& +\hat{f}(\hat{x}, \hat{\bar{x}}, \hat{z}, g, h(\hat{x}), u)-\hat{f}\left(\hat{x}, \hat{\bar{x}}, z^{*}, g, h(\hat{x}), u\right) \\
& +\hat{\bar{f}}(\hat{x}, \hat{\bar{x}}, \hat{z}, \bar{g}, \bar{h}(\hat{\bar{x}}), u)-\hat{\bar{f}}\left(\hat{x}, \hat{\bar{x}}, z^{*}, \bar{g}, \bar{h}(\hat{\bar{x}}), u\right) .
\end{aligned}
$$


Considering $V(t, e), \bar{V}(t, \bar{e}), W(t, e)$ and $\bar{W}(t, \bar{e})$ given in assumption $\mathscr{A} 2)-2$ ) and using $\mathscr{A} 2)-2)-i$ ), we get:

$$
\begin{aligned}
& \frac{d V(t, e, \bar{e})}{d t}=\frac{d V(t, e)}{d t}+\frac{d \bar{V}(t, \bar{e})}{d t} \leq-W(e)-\bar{W}(\bar{e}) \\
& +\left\|\frac{\partial V(t, e)}{\partial e}(f(x, \hat{\bar{x}}, z, u)-f(x, \bar{x}, z, u))\right\| \\
& +\left\|\frac{\partial \bar{V}(t, \bar{e})}{\partial \bar{e}}(\bar{f}(\hat{x}, \bar{x}, z, u)-\bar{f}(x, \bar{x}, z, u))\right\| \\
& +\left\|\frac{\partial V(t, e)}{\partial e}\right\|\left\|\hat{f}(\hat{x}, \hat{\bar{x}}, \hat{z}, g, h(\hat{x}), u)-\hat{f}\left(\hat{x}, \hat{\bar{x}}, z^{*}, g, h(\hat{x}), u\right)\right\| \\
& +\left\|\frac{\partial \bar{V}(t, \bar{e})}{\partial \bar{e}}\right\|\left\|\hat{\bar{f}}(\hat{x}, \hat{\bar{x}}, \hat{z}, \bar{g}, \bar{h}(\hat{\bar{x}}), u)-\hat{\bar{f}}\left(\hat{x}, \hat{\bar{x}}, z^{*}, \bar{g}, \bar{h}(\hat{\bar{x}}), u\right)\right\| .
\end{aligned}
$$

And from assumption $\mathscr{A} 2)-2)-i i i)$, we get:

$$
\begin{aligned}
& \frac{d V(t, e, \bar{e})}{d t} \leq-W(e)-\bar{W}(\bar{e}) \\
& +\alpha_{1} \sqrt{W(e)} \sqrt{\bar{W}(e)}+\alpha_{2} \sqrt{W(e)} \sqrt{\bar{W}(e)} \\
& +\left\|\frac{\partial V(t, e)}{\partial e}\right\|\left\|\hat{f}(\hat{x}, \hat{\bar{x}}, \hat{z}, g, h(\hat{x}), u)-\hat{f}\left(\hat{x}, \hat{\bar{x}}, z^{*}, g, h(\hat{x}), u\right)\right\| \\
& +\left\|\frac{\partial \bar{V}(t, \bar{e})}{\partial \bar{e}}\right\|\left\|\hat{\bar{f}}(\hat{x}, \hat{\bar{x}}, \hat{z}, \bar{g}, \bar{h}(\hat{\bar{x}}), u)-\hat{\bar{f}}\left(\hat{x}, \hat{\bar{x}}, z^{*}, \bar{g}, \bar{h}(\hat{\bar{x}}), u\right)\right\| .
\end{aligned}
$$

From assumption $\mathscr{A} 3)$, we deduce that:

$$
\begin{aligned}
& \frac{d V(t, e, \bar{e})}{d t} \leq-\frac{1}{2}\left(1-\frac{\alpha_{1}+\alpha_{2}}{2}\right)(\sqrt{W(e)}+\sqrt{\bar{W}(\bar{e})})^{2} \\
& +\left(\left(\left\|\frac{\partial V(t, e)}{\partial e}\right\| \lambda_{1}\right)+\left(\left\|\frac{\partial V(t, \bar{e})}{\partial \bar{e}}\right\| \lambda_{2}\right)\right)\left\|\hat{z}-z^{*}\right\| .
\end{aligned}
$$

And finally, from assumption $\mathscr{A} 1)-i i)$, we get:

$$
\begin{aligned}
& \frac{d V(t, e, \bar{e})}{d t} \leq-\frac{1}{2}\left(1-\frac{\alpha_{1}+\alpha_{2}}{2}\right)(\sqrt{W(e)}+\sqrt{\bar{W}(\bar{e})})^{2} \\
& +\left(\left(\left\|\frac{\partial V_{1}(t, e)}{\partial e}\right\| \lambda_{1}\right)+\left(\left\|\frac{\partial V_{2}(t, \bar{e})}{\partial \bar{e}}\right\| \lambda_{2}\right)\right) \sigma^{-1}\|\varphi(\hat{x}, \hat{\bar{x}}, \hat{z})\| .
\end{aligned}
$$

From (13), we have $\lim _{t \rightarrow \infty}\|\varphi(\hat{x}(t), \hat{\bar{x}}(t), \hat{z})\|=0$ and using

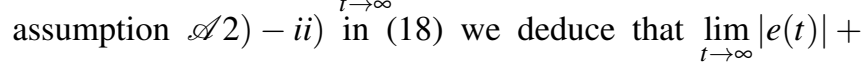
$|\bar{e}(t)|=0$.

From assumption $\mathscr{A} 1)-i i),(\|\hat{z}-z\|) \leq \sigma^{-1}\|\varphi(\hat{x}, \hat{\bar{x}}, \hat{z})\|$ where $z$ is the solution of: $\varphi(x, \bar{x}, z)=0$, using (13) we obtain $\lim _{t \rightarrow \infty}\|\hat{z}(t)-z(t)\|=0$.

\section{EXPLICIT OBSERVER FOR IMPLICIT SYSTEMS WITH TRIANGULAR STRUCTURE}

The first step in the design of the observer (9) is the synthesis of the gain functions $g$ and $\bar{g}$. Many normal forms which characterized some subclasses of multi-output uniformly observable systems together with their associated observer syntheses have been proposed in the literature, see for instance: [11], [3], and [19]. Now, let us come back to the structures (5) and (6). Referring to $(\mathbf{C 1}, \mathbf{C 2})$, we remark that the functions $f$ and $\bar{f}$ have triangular structures with respect to $(h(x), x)$ and $(\bar{h}(\bar{x}), \bar{x})$. In this case, a constant gain $g=K_{\theta}$ (i.e. $\mathrm{G}(\mathrm{g}, \mathrm{u})=0$ ) respectively $\bar{g}=\bar{K}_{\theta}$, can be designed as stated in [3]. As can be seen, system (5) and system (6) have the same structure. Therefore, the same observer structure will be used. Now, by considering the triangular structure of (5) given by C1), C2) and C3) and assuming that the map $(x, \bar{x}, z) \rightarrow(x, \bar{x}, \varphi(x, \bar{x}, z))$ satisfies the assumption $\mathscr{A} 1$ ), we deduce that for $i=1,2$ and $1 \leq k \leq n_{i}$; there exist smooth functions $\gamma_{k}^{i}$ (their analytic expressions are generally unknown) such that the solution $z$ of $\varphi(x, \bar{x}, z)=0$ is given by:

$$
\left\{\begin{aligned}
z_{1}^{i} & =\gamma_{1}^{i}\left(x_{1}^{i}, \bar{x}_{1}^{i}, x_{2}^{i}, \bar{x}_{2}^{i}\right) \\
z_{k}^{i} & =\gamma_{k}^{i}\left(x_{k-1}^{i}, \bar{x}_{k-1}^{i}, x_{k}^{i}, \bar{x}_{k}^{i}, x_{k+1}^{i}, \bar{x}_{k+1}^{i}\right) \quad 1<k<n_{i} \\
z_{n_{i}}^{i} & =\gamma_{n_{i}}^{i}(x, \bar{x}) .
\end{aligned}\right.
$$

In the sequel, we use the same notation as above. We set $F_{k}^{i}(x, \bar{x}, u)=f_{k}^{i}\left(x, \bar{x}, \gamma_{k}^{i}(x, \bar{x}), u\right)$, and $F^{i}=\left[F_{1}^{i}, \ldots, F_{n_{i}}^{i}\right]^{T}, F=$ $\left[\begin{array}{ll}F^{1^{T}} & F^{2^{T}}\end{array}\right]^{T}$, then system (5) can be rewritten:

$$
\left\{\begin{array}{l}
\dot{x}(t)=F(x(t), \bar{x}(t), u(t)) \\
y_{1}(t)=C x(t) .
\end{array}\right.
$$

Moreover $F(x, \bar{x}, u)$ is a $\mathscr{C}^{1}$ function of triangular form and satisfy the following hypothesis.

H1) For every $(x, \bar{x}, u), \rho_{1} \leq \frac{\partial F_{j}^{i}}{\partial x_{j+1}^{i}}(x, \bar{x}, u) \leq \rho_{2}$, for some constants $\rho_{1}>0, \rho_{2}>0$.

H2) $F$ is a global Lipschitz function w.r.t. $x$, $\exists c>0 ; \forall u \in U ; \forall x, x^{\prime},\left\|F(x, \bar{x}, u)-F\left(x^{\prime}, \bar{x}, u\right)\right\| \leq c\left\|x-x^{\prime}\right\|$.

In [3] the authors gave a constant gain observer for systems of the form (19) with $\bar{x}$ as a known variable. Based in this result, a constant high gain observer can be constructed for system (19) as follows:

$$
\left\{\begin{array}{l}
\dot{x}^{1}(t)=F^{1}(\hat{x}(t), \bar{x}, u(t))-r_{1} \Delta_{\theta^{\delta_{1}}} S_{1}^{-1} C_{1}^{T}\left(C_{1} \hat{x}^{1}-x_{1}^{1}\right) \\
\dot{x}^{2}(t)=F^{2}(\hat{x}(t), \bar{x}, u(t))-r_{2} \Delta_{\theta^{\delta_{2}}} S_{2}^{-1} C_{2}^{T}\left(C_{2} \hat{x}^{2}-x_{1}^{2}\right),
\end{array}\right.
$$

where $r_{k} \geq 1 \quad(k=1,2)$ is a positive constant tuning parameter of the observer, $C_{k}=[1, \ldots, 0]$ is a vector of dimension $n_{k} ; \delta_{1}>0, \delta_{2}>0$ satisfy: $\frac{2 l-3}{2 n-2 l+3} \delta_{1}<\delta_{2}<$ $\frac{2 l-1}{2 n-2 l+3} \delta_{1}, \theta>0$ and $\Delta_{\theta} \delta_{i}$ is a $\left(n_{k} \times n_{k}\right)$ diagonal matrix $\Delta_{\theta} \delta_{k}=\operatorname{diag}\left(\theta^{\delta_{k}}, \theta^{2 \delta_{k}}, \ldots, \theta^{n_{i} \delta_{i}}\right)$, and finally $S_{k}$ is an $n_{k} \times n_{k}$ constant SPD matrix of the form:

$$
S_{k}=\left(\begin{array}{ccccc}
s_{11} & s_{12} & 0 & & 0 \\
s_{12} & s_{22} & \ddots & & \vdots \\
0 & \ddots & & \ddots & 0 \\
\vdots & & & \ddots & s_{k-1 k} \\
0 & \ldots & 0 & s_{k-1 k} & s_{k k} .
\end{array}\right)
$$

To assure the exponential convergence of the above observer, the matrix $S_{k}$ is chosen to satisfy the following condition (detail of the proof see [3]):

$$
\forall t \geq 0, \quad A_{k}^{T}(t) S_{k}+S_{k} A_{k}(t)-\rho C_{k}^{T} C_{k} \leq-v I_{k},
$$

where $A_{k}(i, j)=a_{i}^{k}(t)$ for $j=i+1$ and $A_{k}(i, j)=0$ for $j \neq i+$ 1 such that $\rho_{1} \leq a_{i}(t) \leq \rho_{2}$, and $\rho_{1}, \rho_{2}$ are positive constants. Now, let $\Omega_{0}$ be a tubular neighborhood of $\mathscr{M}$ given by the assumption $\mathscr{A} 1$ ). In order to show that system (23) forms an observer for system $(5,6)$, the following assumption is used: H3): $f$ and $\bar{f}$ is a global Lipschitz functions on $\Omega_{0}$, namely: $\exists\left(c^{\prime}, \bar{c}^{\prime}\right)>0 ; \forall u \in U ; \forall(x, \bar{x}, z),\left(x^{\prime}, \bar{x}^{\prime}, z^{\prime}\right) \in \Omega_{0}$, $\left\|f(x, \bar{x}, z, u)-f\left(x^{\prime}, \bar{x}^{\prime}, z^{\prime}, u\right)\right\| \leq c^{\prime}\left\|(x, \bar{x}, z)-\left(x^{\prime}, \bar{x}^{\prime}, z^{\prime}\right)\right\|$. 
$\left\|\bar{f}(x, \bar{x}, z, u)-\bar{f}\left(x^{\prime}, \bar{x}^{\prime}, z^{\prime}, u\right)\right\| \leq \bar{c}^{\prime}\left\|(x, \bar{x}, z)-\left(x^{\prime}, \bar{x}^{\prime}, z^{\prime}\right)\right\|$.

Now, we can state the main result of this section:

Proposition 1: Under assumption $\mathscr{A}_{2}$ ) and hypotheses H1), H3), the following system:

$$
\left\{\begin{aligned}
\dot{\hat{x}}= & f(\hat{x}, \hat{\bar{x}}, \hat{z}, u)+K_{\theta}\left(\hat{y}_{1}-y_{1}\right) \\
\dot{\hat{x}}= & \bar{f}(\hat{x}, \hat{\bar{x}}, \hat{z}, u)+\bar{K}_{\theta}\left(\hat{y}_{2}-y_{2}\right) \\
\dot{\hat{z}}(t) & =-\left(\left.\frac{\partial \varphi}{\partial z}\right|_{(\hat{x}, \hat{z}, \hat{x})}\right) \\
& \times\left(\left[\left.\frac{\partial \varphi}{\partial x}\right|_{(\hat{x}, \hat{z})},\left.\frac{\partial \varphi}{\partial \bar{x}}\right|_{(\hat{x}, \hat{z})}\right][\dot{\hat{x}}, \dot{\hat{x}}]^{T}+\Lambda \varphi(\hat{x}, \hat{\bar{x}}, \hat{z})\right)
\end{aligned}\right.
$$

where $K_{\theta}=\operatorname{diag}\left(-r_{1} \Delta_{\theta^{\delta_{1}}} S_{1}^{-1} C_{1}^{T},-r_{2} \Delta_{\theta} \delta_{2} S_{2}^{-1} C_{2}^{T}\right)$

$$
\bar{K}_{\theta}=\operatorname{diag}\left(-\bar{r}_{1} \bar{\Delta}_{\theta^{\delta_{1}}} \bar{S}_{1}^{-1} \bar{C}_{1}^{T},-\bar{r}_{2} \bar{\Delta}_{\theta^{\delta_{2}}} \bar{S}_{2}^{-1} \bar{C}_{2}^{T}\right),
$$

forms an explicit observer for system (7).

The proof of this proposition is accomplished by applying Theorem 1 of the above section, where $\hat{f}(\hat{x}, \hat{\bar{x}}, \hat{z}, h(\hat{x}), g, u)=f(\hat{x}, \hat{\bar{x}}, \hat{z}, u)+K_{\theta}\left(\hat{y}_{1}-y_{1}\right) \quad$ and $\hat{\bar{f}}(\hat{x}, \hat{\bar{x}}, \hat{z}, \bar{h}(\hat{\bar{x}}), \bar{g}, u)=\bar{f}(\hat{x}, \hat{\bar{x}}, \hat{z}, u)+\bar{K}_{\theta}\left(\hat{y}_{2}-y_{2}\right)$.

To do, it suffices to show that assumptions $\left.\mathscr{A}_{2}\right)$ and $\mathscr{A}_{3}$ ) hold. Setting $V(t, e)=e^{T} \Delta_{\theta} S \Delta_{\theta} e$ and $\bar{V}(t, \bar{e})=\bar{e}^{T} \bar{\Delta}_{\theta} \overline{S \Delta}_{\theta} \bar{e}$, so $V$ and $\bar{V}$ are positive definite quadratic functions and do not depends on $t$. Thus condition of assumption $\mathscr{A} 2$ ) -2 ) $-i i$ ) is satisfied. Let us now check the condition $\mathscr{A} 1)-1)-i$ ): By construction, we know that if $(x, \bar{x}, z) \in \mathscr{M},(\hat{x}, \hat{\bar{x}}, \hat{z}) \in \mathscr{M}$, then: $f(x, \bar{x}, z, u)=F(x, \bar{x}, u), \quad \bar{f}(x, \bar{x}, z, u)=\bar{F}(x, \bar{x}, u)$ and $\hat{f}(\hat{x}, \bar{x}, \hat{z}, g, h(\hat{x}), u)=F(\hat{x}, \bar{x}, u)+K_{\theta}\left(h(\hat{x})-y_{1}\right)$, $\hat{\bar{f}}(x, \hat{\bar{x}}, \hat{z}, \bar{g}, \bar{h}(\hat{\bar{x}}), u)=\bar{F}(x, \hat{\bar{x}}, u)+\bar{K}_{\theta}\left(\bar{h}(\hat{\bar{x}})-y_{2}\right)$.

Now since H1), H2) are satisfied for $F(x, \bar{x}, u), \bar{F}(x, \bar{x}, u)$, inequalities (11) and (12) holds. Consequently, $\mathscr{A} 2)-2$ ) i) is satisfied. Assumption $\mathscr{A}_{3}$ )) is a direct consequence of hypothesis H3) and the fact that the gain of the observer is constant and the output is a linear map. In the end it remains to verify the assumption $\mathscr{A} 2$ ) -2 ) - iii) which is the full interconnection condition [20], it is also a direct consequence of hypothesis $\mathbf{H} 3$ ).

\section{SIMULATION RESULTS}

\section{A. Experimental validation of the model ${ }^{1}$}

In this section we will analyse the validity of the controloriented DAE model (1) developed above. It is solved using the Runge-Kutta method combined with the NewtonRaphson optimization algorithm.

As first step of the model validation is the study of the model behaviour when the column is assumed to be stabilized around its steady state. To do so, we consider an experimental configuration of the steady state shown in table 1 . The simulation results of this test are given in Figure 2 which shows the evolution over time of the liquid molar fractions of benzene in the condenser, the boiler and the trays 3 and 9. We note the existence of a negligible transient compared to the initial values, which prove the good accuracy of the model.

\footnotetext{
${ }^{1}$ Authors acknowledge the company Processium for the experimental data.
}

\begin{tabular}{|l|l|}
\hline Tray index: total & $n=18$, \\
side stream and feed trays in con- & $s=6, l=9$ \\
Liquid molar retention in & $N_{1}=20 \mathrm{~mol}$ \\
denser & \\
$\begin{array}{l}\text { Liquid molar retention in boiler } \\
\text { Liquid molar retention in each }\end{array}$ & $N_{18}=20 \mathrm{~mol}$ \\
tray & $N_{i}=8 \mathrm{~mol}$ \\
Liquid and vapour Murphree effi- & $e^{l}=1, e^{v}=1$ \\
ciency & \\
Internal pressure & $P_{T}=760 \mathrm{mmHg}$ \\
Feed flow rate & $F=1.67 \mathrm{~mol} / \mathrm{min}$ \\
Benzene composition in feed rate & $Z_{B e n, l}=0.6$ \\
Toluene composition in feed rate & $Z_{T o l, l}=0.3$ \\
Feed temperature & $T_{l}=379,32 \mathrm{~K}$ \\
Side stream liquid flow rate & $S=0.167 \mathrm{~mol} / \mathrm{min}$ \\
Steady state vapour flow rate & $V_{f}=7.0086 \mathrm{~mol} / \mathrm{min}$ \\
Steady state liquid flow rate & $L=6.0017 \mathrm{~mol} / \mathrm{min}$ \\
Steady state quality of Benzene in & $x_{1,1}=0.95$ \\
top product & \\
Steady state quality of Benzene in & $x_{1,18}=8.87 \times 10^{-5}$ \\
bottom product & \\
Steady state quality of Toluene in & $x_{2,1}=0.05$ \\
top product & \\
Steady state quality of Toluene in \\
bottom product
\end{tabular}

TABLE I

OPERATING POINT AND INITIAL STEADY STATE.

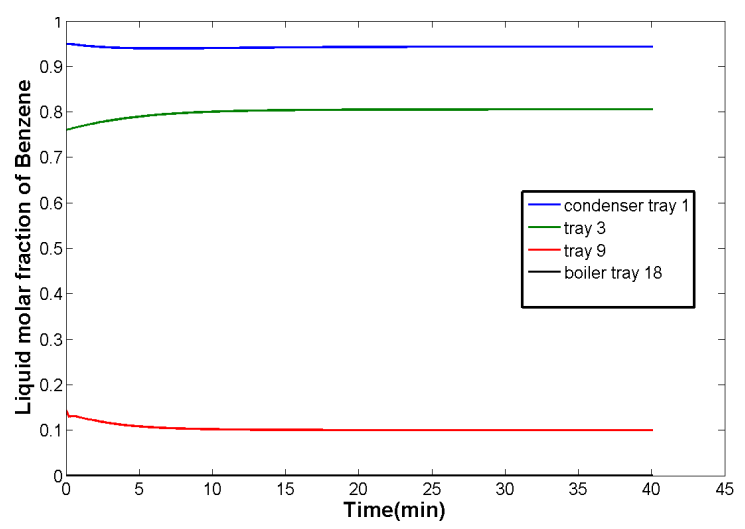

Fig. 2. Response of the dynamic model starting from the experimental steady state, in 4 locations: liquid molar fractions of benzene.

A second step of the model validation is done using experimental data. Figure 3 shows the simulated and experimental steady state profiles of molar fractions of the ternary mixture BTX according to the number of trays. We can note a good agreement.

\section{B. Observer simulation}

To simulate the response of the observer and compare it with the response of the implicit system, we kept the same configuration of the operating point and initial conditions given in the previous part. For the observer we initialized the estimated states to arbitrary values different from those of model. The values of the observer parameters $r$ and $\theta$ are set to 1 and 2.8 respectively. The output measurements $y_{1}(t)$ and $y_{2}(t)$ feature a zero mean Gaussian noise of $5 \%$. The performances of the observer are illustrated in the figures 4 and 5: in spite of the errors in the initial condition, 


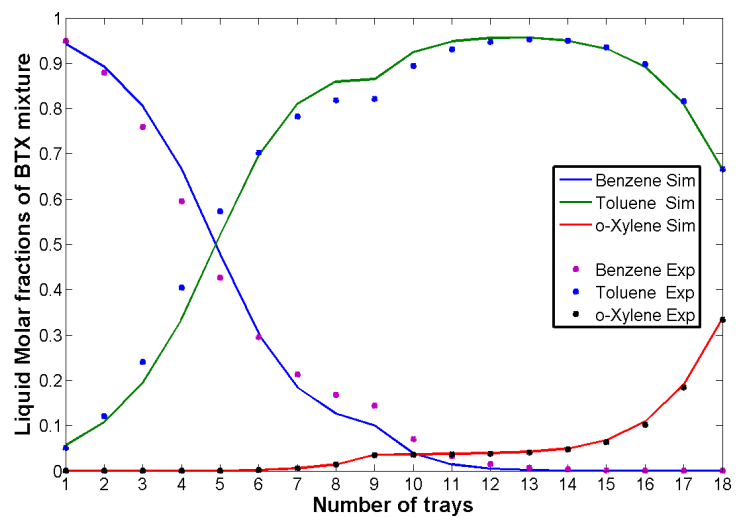

Fig. 3. Final molar fraction profiles: simulation and experimental.

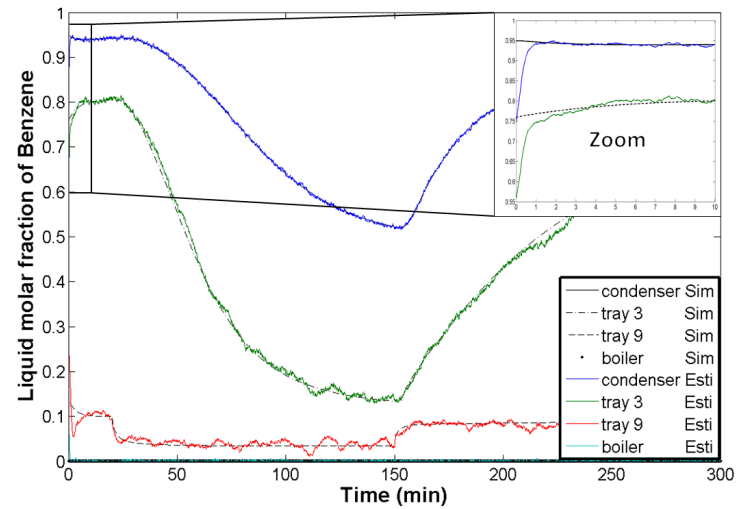

Fig. 4. Four true and estimated benzene molar fractions, in the presence of $5 \%$ noise and initial errors in the estimations, $\theta=2.8$.

noise measurements and the disturbances, the estimated state converge to their dynamic target values, for both the molar fraction and the temperature profiles.

\section{CONCLUSION}

In this work we first developed and validated experimentally a mathematical model (a set of differential algebraic equations) that describes the dynamic of the temperature and component molar fraction profiles of a ternary mixture in a distillation column. The obtained model is an implicit systems fully interconnected based on the triangular structure of the model, an explicit high gain observer is given using bottom and top molar fractions measurement. The observer implementation is simple and requires small computational effort. Furthermore, simulation results demonstrate the good performances achieved by this observer in coping with model nonlinearities and output noise.

\section{REFERENCES}

[1] J.Gmehling, B.Kolbe, M.Kleiber and J.Rarey, Chemical thermodynamics for process simulation, WILEY-VCH, 2012.

[2] R. Baratti, A. Bertucco, A.D. Rold and M. Morbidelli, "A composition estimator for multicomponent distillation columns-development and experimental test on ternary mixtures", Chemical Engineering Science, 1998, vol. 53, no 20, pp. 3601-3612,

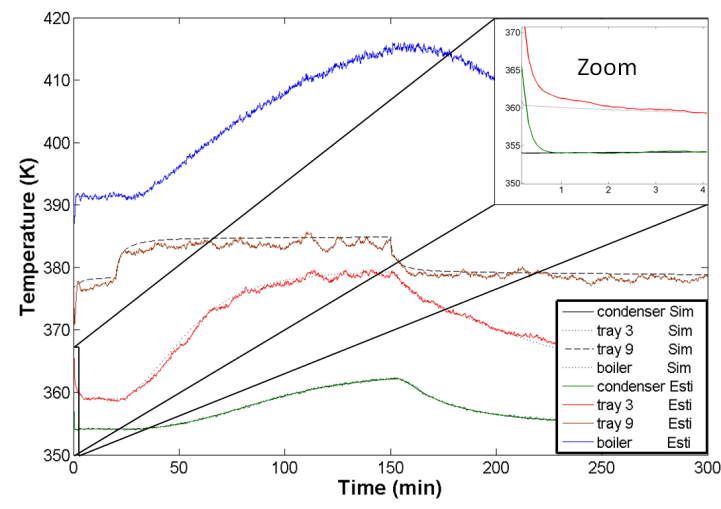

Fig. 5. Four true and estimated temperatures, in the presence of $5 \%$ noise and initial errors in the estimations, $\theta=2.8$.

[3] H.Hammouri, B.Targui and F. Armanet, "High gain observer based on a triangular structure", Int. Journal of Robust Nonlinear Control, 2002, vol. 12, no 6, pp.497-518.

[4] P.C. Muller and M. Hou, "On the observer design for descriptor systems", IEEE Transactions on Automatic Control, 1993, vol. 38, no 11 , pp. 1666-1671.

[5] M. Darouach and M. Boutayeb, "Design of Observers for Descriptor Systems", IEEE Transactions on Automatic Control, 1995, vol. 40, no 7, pp. 1323-1327.

[6] M. Darouach, M. Zasadzinski and M. Hayar, "Reduced order observer design for descriptor systems with unknown inputs", IEEE Transactions on Automatic Control, 1996, vol. 41, no 7, pp.1068-1072.

[7] T. Boukhobza, F. Hamelin and D. Sauter, "Observability of structured linear systems in descriptor form: A graph-theoretic approach", Automatica, 2006, vol. 42, no 4, pp.629-635.

[8] R. Nikoukhah, A.S. Willsky and B.C. Levy, "Kalman filtering and Riccati equations for descriptor systems", IEEE Transactions on Automatic Control, 1992, vol. 37, no 9, pp. 1325-1342.

[9] A.J. Krener and W. Respondek, "Nonlinear observers with linear error dynamics", SIAM Journal on Control and Optimization, 1985, vol. 23, no 2, p. $197-216$.

[10] H. Hammouri and J.P. Gauthier, "Bilinearization up to output injec tion”, Systems and Control Letters, 1988, vol. 11, no 2, pp.139-149.

[11] J.P. Gauthier and I. Kupka, "Observability and observers for nonlinear systems", SIAM Journal Control and Optimization, 1994, vol. 32, no 4, pp. $975-994$.

[12] I.B. Tjoa and L.T. Biegler, "Simultaneous solution and optimization strategies for parameter estimation of differentialalgebraic equation systems", Industrial and Engineering Chemistry Research, 1991, vol. 30, no 2, pp. 376-385.

[13] V.M. Becerra, P.D. Roberts and G.W. Griffiths, ”Applying the extended Kalman filter to systems described by nonlinear differential algebraic equations", Control Engineering Practice, 2001, vol. 9, no 3, pp. 267 281.

[14] R. Nikoukhah, "A new methodology for observer design and implementation", IEEE Transactions on Automatic Control, 1998, vol. 43 , no 2, pp. 229-234.

[15] J. Aslund and E. Frisk, "An observer for non-linear differentialalgebraic systems", Automatica 42, 2006, vol. 42, no 6, pp. 959-965.

[16] G. Zimmer and J. Meier, "On observing nonlinear descriptor systems", System and Control Letters, 1997, vol. 32, no 1, pp. 43-48.

[17] J. Gmehling and U. Onken, Vapor-liquide equilibrium data colection, Aromatic Hydrocarbons, DACHEMA Chemistry Data Series, vol.1 Part 7a (in conjunction with Part 7b), 1985.

[18] H. Hammouri and M. Nadri, "An observer design for a class of implici systems", Systems and Control Letters, 2013, vol. 62, no 3, pp. 256261.

[19] H. Hammouri and M. Farza, "Nonlinear observers for locally uniformly observable systems", Control, Optimization and Calculus of Variation; ESAIM COCV, 2003, vol. 9, pp. 353-370.

[20] G. Besançon and H. Hammouri, "On observer design for interconnected systems", Journal of Mathematical Systems, Estimation, and Control, 1997, vol. 8, No. 3, pp. 1-25. 